\title{
Public Opinions Impacts on Cryptocurrency Valuation: A View from Behavioral Finance Perspective
}

\author{
Andrey A. Gusev ${ }^{1,2, *}$ \\ ${ }^{1}$ Department of corporate finance and corporate governance, Finance University under the Government of \\ the Russian Federation, Moscow, 49 Leningradsky Prospect, Moscow, 125993, Russia \\ ${ }^{2}$ IBS Department, The Russian Presidential Academy of National Economy and Public Administration \\ (RANEPA), 82 Prospect Vernadskogo, Moscow, 119571, Russia
}

\begin{abstract}
Many fintech start-ups participant companies and cryptocurrencies have experienced phenomenal growth in value during the past several years. Many specialists would like to know the reasons for such success. In this article we investigate the predictive power of public opinions. This is one of the few works that using quantitative analysis connects social media and internet users' activities with cryptocurrency valuations.
\end{abstract}

Keywords: Fintech, Valuation, Cryptocurrency, Cryptoeconomy, Behavioral Finance, Predictive Power.

\section{INTRODUCTION}

Wisdom of crowds is a term used to embrace the fact that the mean collective opinion of individuals, in many cases, can be used to make rather accurate forecasts and approximations. This principle has been widely adopted and investigated by the scientific community in the field of finance. It involves analyzing messages from market participants or market influencers, and comparing them with future asset returns, volatility or trading volume. The influence of social information was very significantly studied by Fama and Fisher (1969), Grossman and Stiglitz (1976), Bikhchandani (1992). Growth of social research is supported through the development of IT tools for gathering and storing big data.

This paper aims to analyse the interdependence between social signals and prices of cryptocurrencies in the digital economy.

Prior to our analysis we performed stationarity tests of the time-series, revealing that these variables are integrated of order 1: the time-series cannot be assumed to be stationary, but the first differences are stationary and can be used in regression models.

This paper is structured is as follows: in Section II we make short literature analysis, in Section III we discuss the methods and algorithms used, in Section IV we describe the data and its sources and show our results and Section $V$ holds the conclusion.

*Address correspondence to this author at the Department of Corporate Finance and Corporate Governance, Finance University under the Government of the Russian Federation, Moscow; 49 Leningradsky Prospect, Moscow, 125993, Russia; Tel: 7-499-943-9495; E-mail: gusevaa@yandex.ru

\section{LITERATURE REVIEW}

Fintech has the biggest impact on corporate finance and financial institutions activities. Radical simplification, digitalization of processes and rationalization offer non-bank competitors an unprecedented cost advantage. Distributed ledger technologies (Bitcoin) may enable cost-effective storage and rapid clearing and settlement of transactions in the banking back office. Separate digital banks under separate brands may be a possible solution for traditional banks to be able to experiment with new technologies. To build a successful business model is the most important task for financial institutions. To answer this challenge, the financial sector should fundamentally change its culture and strategy for success.

Fintech start-ups focus on developing analytical tools for financial institutions (Gusev, 2017). They are apt to transform customer experience targeting points of interaction between the client and the bank, their main advantage being seamless service and flexibility.

Start-ups choose to combine technology with a certain banking process blend fintech innovations into their business and operating models, launch attractive new services, reach underserved segments, and differentiate the client experience-at competitive price parameters s and lower operating costs (Gusev, 2018).

Capital optimization is a traditional function in running a bank. Al tools based on mathematical concepts, big data and computing power increase efficiency, accuracy and speed of capital optimization. Trading generates large quantities of data necessary for learning purposes. Machine learning can be used 
by exchanges to determine when members' trading account positions may have increased risk and warrant intervention. Large trading firms use risk management techniques based on big data analysis which enable them to manage risks and optimize their use of capital by centralizing risks that arise from various parts of their business.

Effects of $\mathrm{Al}$ on financial institutions are profound and perceived throughout the sector. Machine learning enhances processing of various operations thus increasing revenues and reducing costs. However, experts point out that Al may create "black boxes" in decision-making and complicate issues in tail events (Franco, 2014). There may be a lack of clarity and responsibility across the financial system. Al and machine learning may miss new types of risks because they could potentially "overtrain" on past events. The lack of transparency around applications may be problematic for both institutions and regulators as a result of uncertainty in the governance structure.

In contrast to $\mathrm{Al}$, blockchain technology remains largely unregulated and draws many harbingers of imminent failure. It remains the subject of growing attention to a great extent due to hype and legendary volatility. Most cryptocurrencies rise and fall during the trading day by 50 to $100 \%$. It presents a certain problem to recognize which factors influence the exchange rate jumps.

In their book, Akerlof and Schiller highlight the most important factors used in decision-making by market participants and explain how these factors affect market turbulence (Akerlof and Schiller, 2009). The irrational beginning mentioned by J.M.Keynes in the book "General theory of Employment, Interest and Money" was not included in the simplified models of the later years, thus ignoring the influence of confidence (sentiment) in the market, a sense of justice, abuse, monetary illusion and the spread of rumors among the participants. Difficulties in recording these phenomena and including them in the calculations reduce the possibility of forecasting for macroeconomic models based on market statistics. One of the reasons for the crises of dot-com, real estate and other Schiller calls ignoring irrational behavioral factors. The economy is a lot of illogical and disordered and this characterizes the behavior of people in uncertainty. Schiller comes to a conclusion that crises are caused by "changes in our thinking" that goes to a section with the standard economic theories (Shiller, 2016). Illusions forced people to invest on the eve of 2008 in the real estate on which from the rational point of view there couldn't be demand, illusion have caused the last peak in the market of cryptocurrencies in 2017. Such fluctuations are a consequence of limited rationality (Thaler, 2015) and a number of delusions inherent in the person:

- A basis of the economic relations is the trust, confidence in tomorrow, expectations

- Ideas of justice influence decision-making at the prices, the wage level, consent with terms of the contract

- Dishonesty and opportunities for swindles and the frauds existing in economy

- Monetary illusion (a difference between nominal or real rates)

- A story about our life and people around, extended by participants of the market

Taking into account postulates of behavioral economy the concept "superheated economy" means inaction of the regulator in the conditions of the increased trust, adoption by participants of excess risks and investment into obviously unprofitable projects, moral risk (when performers pursue the interests, and risks lie on other participants of the contract), abnormally high level of consumption and distribution of stories about success of ventures. Such speculative fever can cover a certain sector of economy, for example, the cryptocurrency market in 2017 and rise of ICO financing in 2016-2017.

\section{THE MODEL}

The cryptoeconomy is growing at an unprecedented speed. Recent price hikes in 2017 attracted significant attention from mass media and as a consequence caused a cryptocurrency bubble (another "gold rush" of the 21st century). In the cryptoeconomy there are around a thousand financial instruments available to unqualified investors. From this point of view any cryptocurrency system may be viewed as a social experiment. Highly volatile speculative instruments attract traders with considerable risk acceptance. Social interactions strengthen the trend (Fuchs, 2015).

Our hypothesis is that this growth is enhanced by online interactions of individual users. To find the interdependence between communications in social media and cryptocurrency exchange rates, we use evidence gathered from social blogs and the number of inquiries from Google Trends and Wikipedia statistics. 


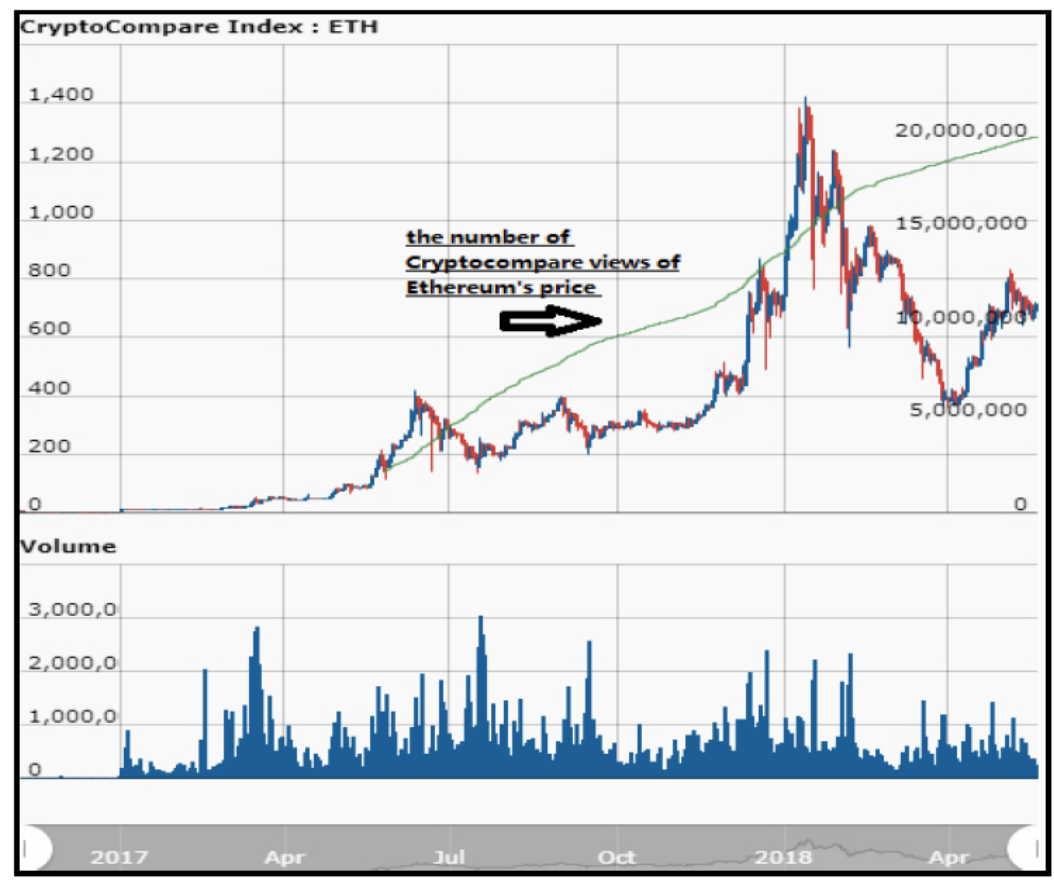

Figure 1: ETC exchange rate.

Cryptocurrencies are traded for fiat and other cryptocurrencies at public Internet exchanges. Currently the biggest cryptoexchanges by volume are Bitfinex, HitBTC, Bitstamp, Coinbase, Kraken, itBit, Neraex, CoinsBank, etc. If we take BTC as an example, it is clear that most of its mined volume is stored in cryptowallets and a very limited number is actually traded in exchanges which makes the market really thin and volatile. Most of the volume is traded in Japanese yen (56\%, 154136 BTC), 17\% in US dollars, $3 \%$ in euros (7000 BTC). The deflationary character of most cryptocurrencies motivates certain modes of behavior (Kolb and Baker, 2010). As the emission is limited by the algorithm, users are motivated to accumulate and store coins. Out of the mined 17000 000 BTC only around 250000 BTC are traded per month (data from cryptocompare.com).

In the cryptoeconomy, the fixed supply and predictable scarcity create a strong link between user adoption, public interest and price (Kelly, 2014). In this research we aim to quantify socio-economic signals to provide an analytical perspective on the relationship between tokens and the social interactions and activities of Internet users.

\section{RESULTS}

\section{Data}

We have gathered 3 datasets of social variables from open sources: Google Trends provided normalized data for social trends, the number of Wikipedia inquiries show the level of interest for cryptocurrencies, Yandex.Blogs filters help to define the number of messages in the social media.

We retrieved the daily number of views from cryptocurrency pages on the English Wikipedia (Ripple, Ethereum, Litecoin) by using the JSON interface of http://stats.grok.se for 3 consecutive years. We gathered the relative volume of blog statistics for the "BTC" search term for 2 consecutive years. We retrieved search volume from Google Trends for two yearly periods $(2016,2017)$. We downloaded the data on miners' rewards and the number of IP addresses from the website blockchain.info.

Another question that had to be solved was determining the period of analysis. As most cryptocurrencies are short-lived, it is nearly impossible to collect a meaningful basket so the approach used in this article is to focus on the period of intense communication at the height of the cryptocurrence bubble. Thus, it is mostly limited by the previous year 2017 , analysed on a daily, weekly and monthly basis.

\section{Results}

The following illustration of the trend shows a definite correlation between the level of user interest from Google Trends and the exchange rate of BTC: the higher the price, the higher the interest score from Google. 


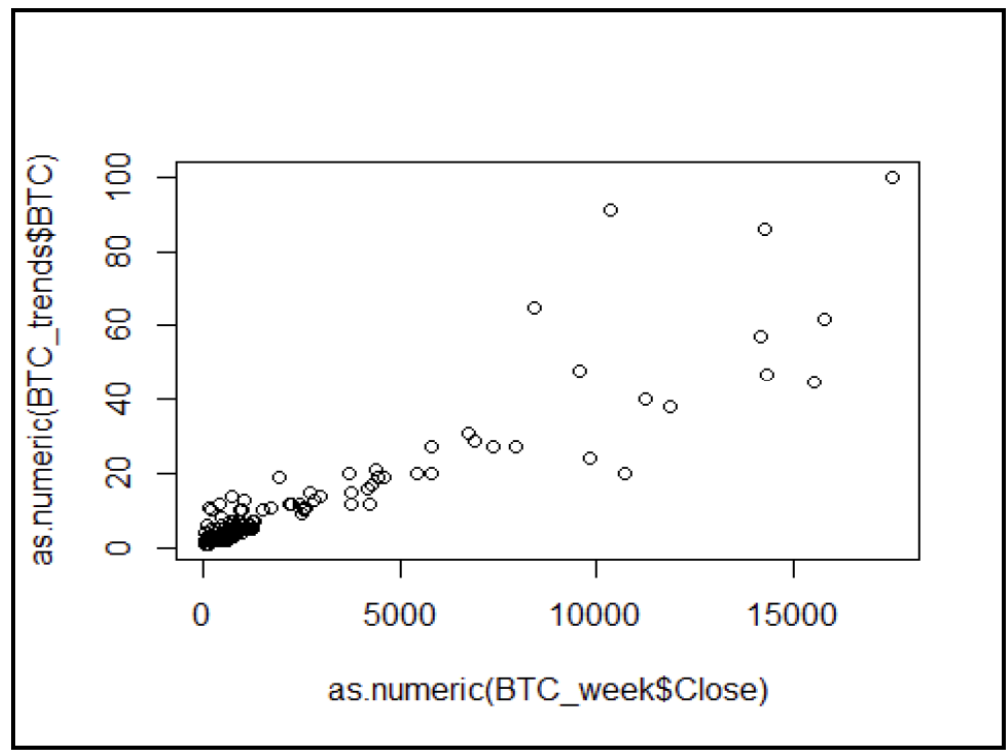

Figure 2: Interdependence between the BTC price (Close) and Google's evaluation of users' interest.

The next figure presents interdependence between the token of a startup and its blockchain platform (in this case, Ethereum). The news component in the price of the cryptocurrency drives up the exchange rate of tokens based on ERC20 standard.

Cindicator is a startup focused on hybrid intelligence for effective asset management. The idea is to provide a platform where community members could make predictions for a reward and Al could be employed to process these forecasts to produce advice for investors. The aim is to support cryptocurrency investors with predictive analytical tools to measure expectations of the market participants, to see market opportunities tapping into the wisdom of the crowd.

In Figure $\mathbf{4}$ we present the regression function explaining the influence of ETH on CND:

As we see the percentage of explained regression $3.6 \%$ and the coefficient (ETH) equals 0.57 . it means that the model can be improved by adding more variables to the function.

The next dataset does not prove strong interdependence between the variable Google Trends

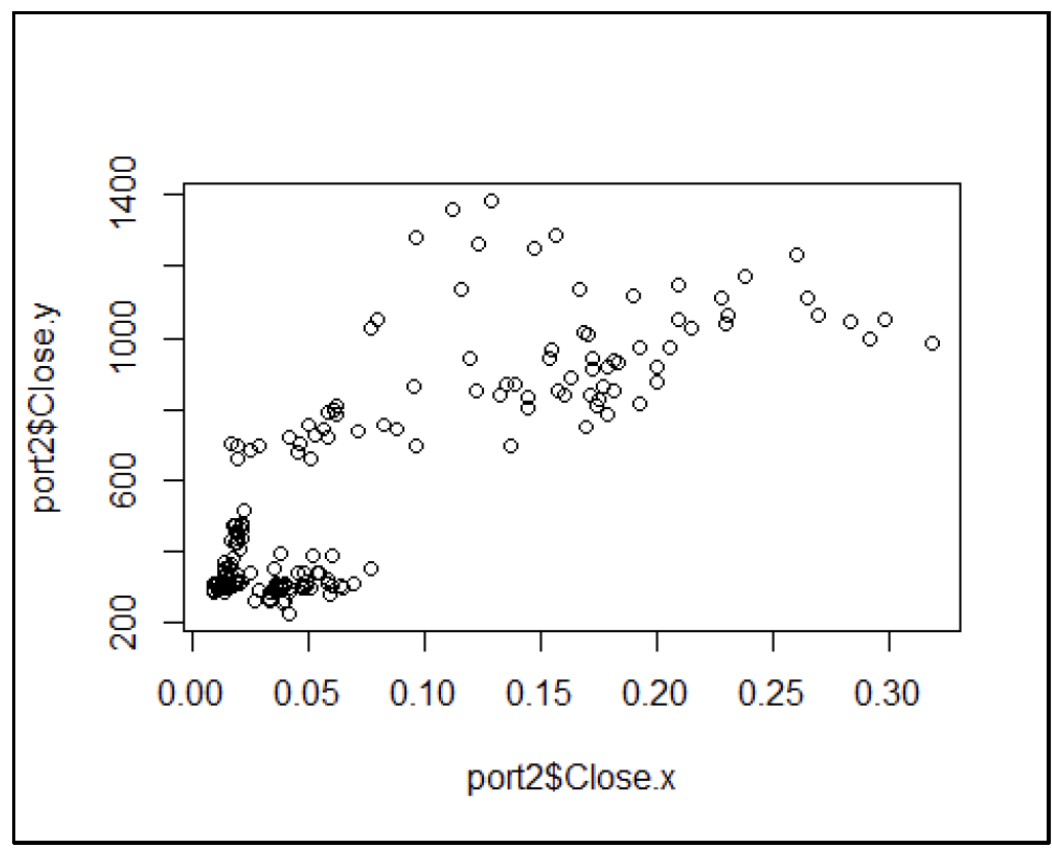

Figure 3: Visualization of the trend Cindicator's token: CND (Close $\mathrm{x}) \sim$ ETC (Close y). 


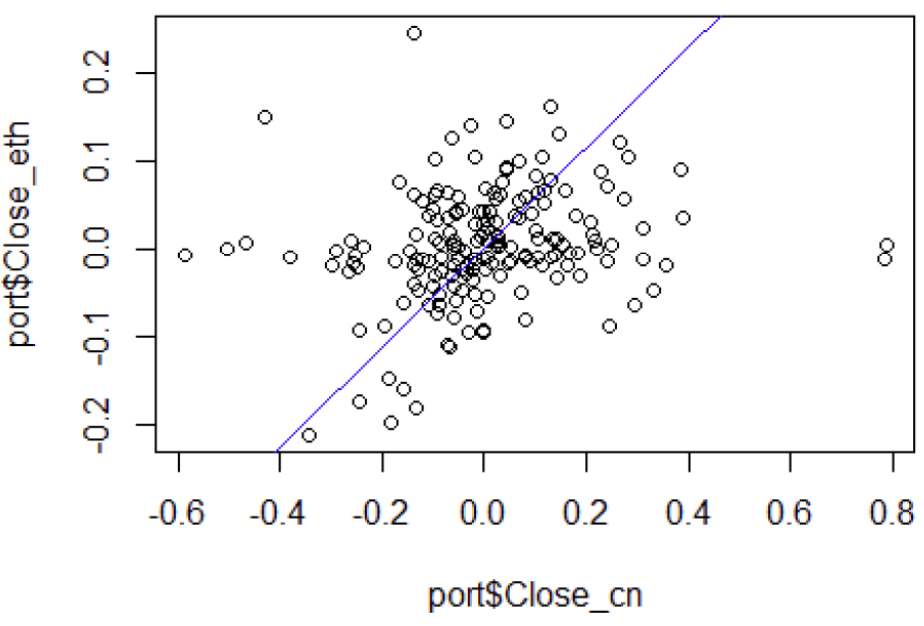

Figure 4: The regression function showing the influence of ETH on CND.

(cindicator) and the CND exchange rate. Beta is close to zero, which leads to the conclusion that users interactions have no immediate effect on the token's profitability but enhance the general trend and provide a long-term effect on its acceptance by the community.

Model 1: Price_CND $=-0.012+0.046$ * Google_TR

Model 2: Price_NEO $=0.018+0.33 *$ NEO_Google_tr $(\alpha<5 \%, R 2=1 \%)$

Model 3: Price_Waves $=-0.011+0.07$ *

Waves_Google_tr $(\alpha<1 \%, \mathrm{R} 2=19 \%)$

Model 4: Price_Binance $=0.014+0.13$ *

BNB_Google_tr $(\alpha<1 \%, \mathrm{R} 2=11 \%)$

Wikipedia datasets provided us with similar results. The percentage of explain regression remains low and sensitivity of tokens to the number of inquiries (public interest) is close to zero.

Ripple model: $\mathrm{XRP}=0.001115+0.024072 *$ Wiki $(\alpha<0.01$, $\mathrm{R} 2=0.006$ )

Ethereum model: ETC $=0.005516+0.033897^{*}$ Wiki $(\alpha<0.01, \mathrm{R} 2=0.007)$

Litecoin model: $L T C=0.003572+0.069616^{*}$ Wiki $(\alpha<0.001, \mathrm{R} 2=0.05)$

The regression function generated by blogs statistics supports the previous tendency: BTC profitability has weak correlation with social networks.

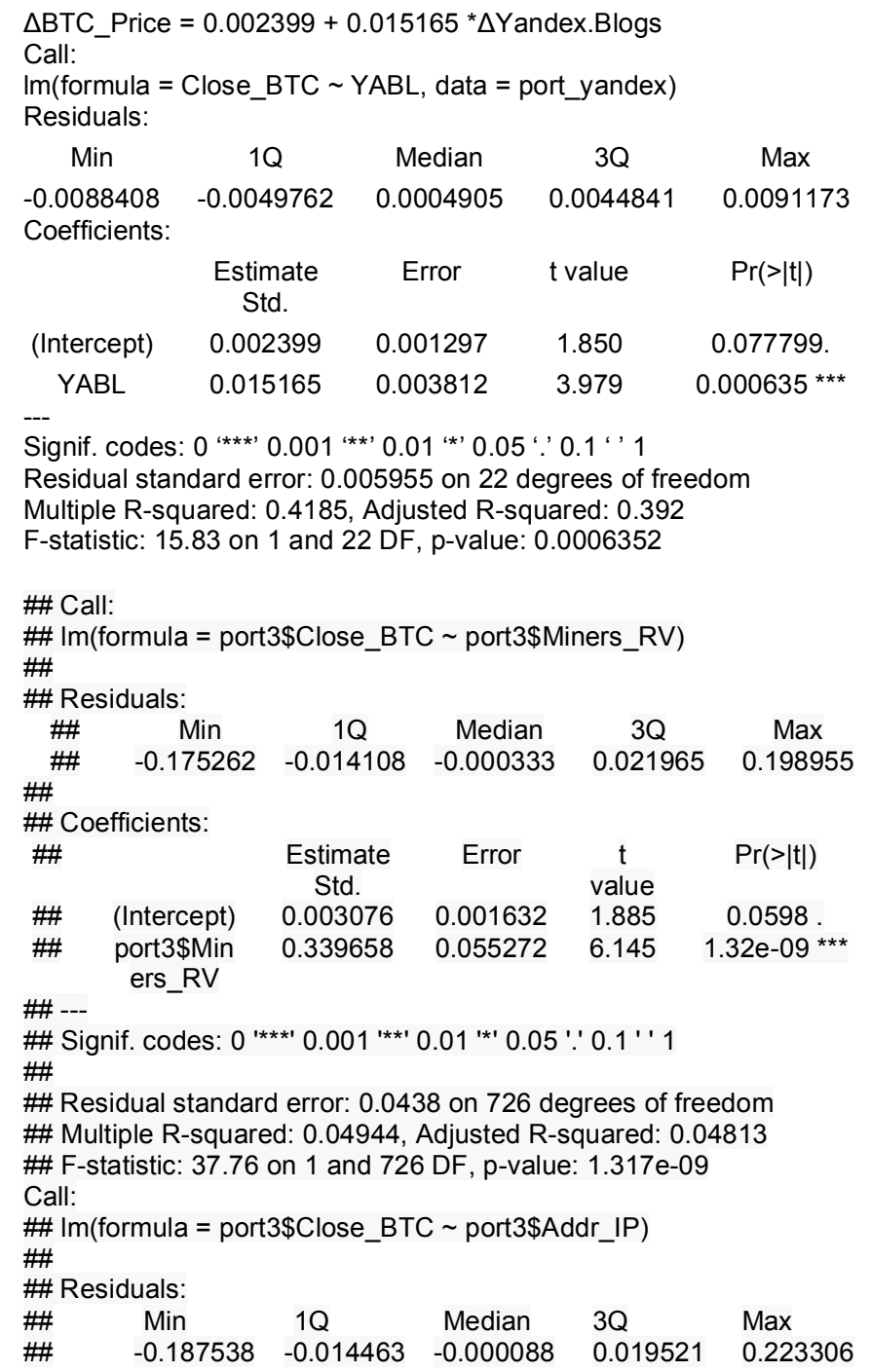




\section{Log miners revenue for $\mathbf{2 0 1 6 - 1 8}$}

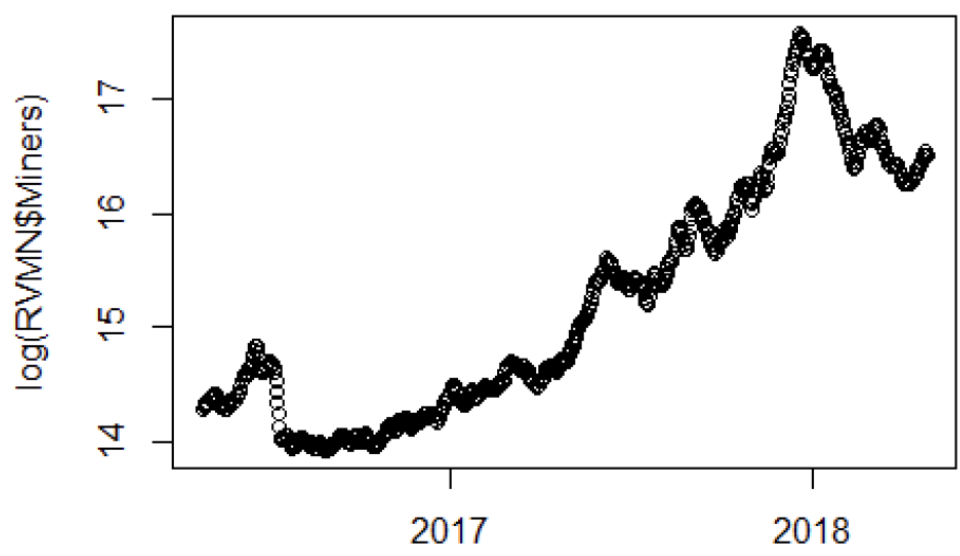

RVMN\$Date

Figure 5: Log miners revenue for 2016-18.

\section{Log new IP Addresses 2016-2018}

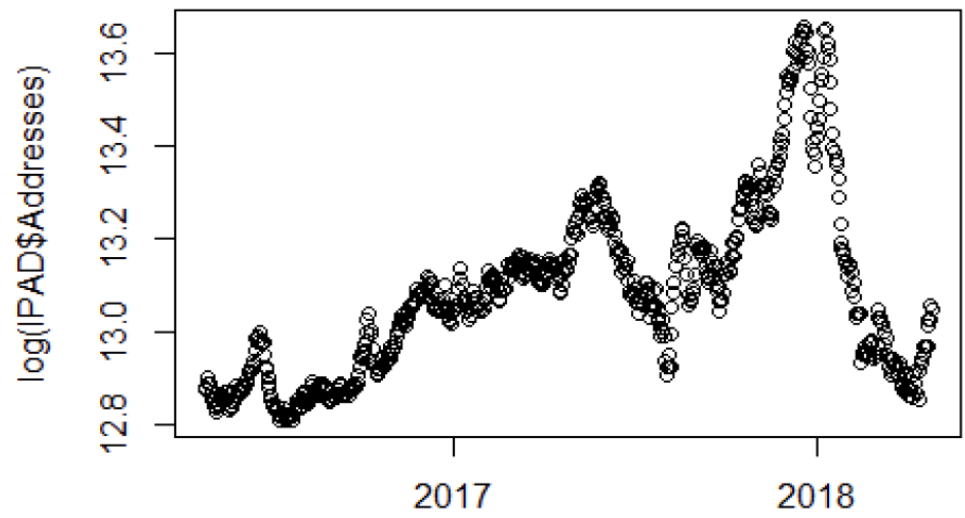

IPAD\$Date

Figure 6: Log new IP Adresses 2016-18.

\#\#

\#\# Coefficients:

\#\# Estimate Error t value $\operatorname{Pr}(>|\mathrm{t}|)$

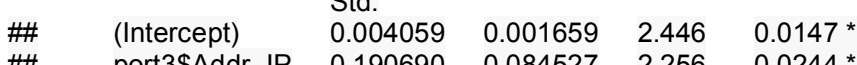

$\begin{array}{llllll}\text { \#\# } & \text { port3\$Addr_IP } & 0.190690 & 0.084527 & 2.256 & 0.0244 \text { * }\end{array}$

\#\# ---

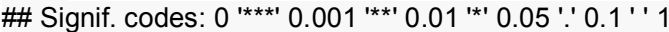

\#\#

\#\# Residual standard error: 0.04476 on 726 degrees of freedom \#\# Multiple R-squared: 0.006961, Adjusted R-squared: 0.005593 \#\# F-statistic: 5.089 on 1 and 726 DF, p-value: 0.02437

As can be concluded from the models with variable "miners' revenue" and "increase in IP addresses", there predictive power is also quite weak and lead to the conclusion that social data can be used to explain longterm effects of the trend rather than immediate impact on profitability.

\section{CONCLUSION}

Even though we managed to produce a sensitivity analysis of tokens and cryptocurrencies, the predictive capabilities are to be enhanced with additional data.

This paper leaves out some questions to be answered in later studies. Cryptocurrency research could be proved more useful. Term frequency could also be used in order to take into account the length of articles. This will be the main focus of our further research.

\section{REFERENCES}

Akerlof G.A. and Robert J. Shiller. "Animal Spirits How Human Psychology Drives the Economy, and Why It Matters for Global Capitalism." Princeton University Press, 2009. 
Bikhchandani S. "A theory of fads, fashion, custom, and cultural change as informational cascades." J. Polit. Econ., 1992, 100, pp. 992-1026.

Fama E., Fisher L. "The adjustment of stock prices to new information." International Economic Review, 1969, 10, pp. 121.

Franco P. "Understanding Bitcoin: Cryptography, Engineering and Economics." John Wiley \& Sons Incorporated, 2014.

Fuchs C. "Culture and Economy in the Age of Social Media." Routledge, 2015.

Grossman S., Stiglitz J. "Information and competitive systems." Am. Econ. Rev. 1976, 246-253.

Gusev A. A. "Value Factors Management to Enhance the Efficiency of Innovative Startups in Russia." Russian Presidential Academy of National Economy and Public Administration (RANEPA). Working Paper № AAAA-A17-117013150012-6, 2017.
Gusev A. A. "Value Creation Practice in Russian Fintech Firms: Analysis." Financial Management, DiS, 2018, 1.

Hitchner J.R. "Financial Valuation: Applications and Models." John Wiley \& Sons Incorporated, 2011.

Kelly B. "The Bitcoin Big Bang: How Alternative Currencies Are about to Change the World." John Wiley \& Sons Incorporated, 2014.

Kolb. R.W.; Baker, H. K. "Behavioral Finance: Investors, Corporations, and Markets." John Wiley \& Sons Incorporated, 2010.

Shiller R. J. "Irrational Exuberance." Revised and Expanded Third Edition. Princeton University Press, 2016.

Thaler, R. H. "Misbehaving: The Making of Behavioral Economics." New York: W. W. Norton \& Company, 2015.

Received on 04-06-2018

Accepted on 19-08-2018

Published on 12-11-2018

DOI: https://doi.org/10.6000/1929-7092.2018.07.63

(C) 2018 Andrey A. Gusev; Licensee Lifescience Global.

This is an open access article licensed under the terms of the Creative Commons Attribution Non-Commercial License (http://creativecommons.org/licenses/by-nc/3.0/) which permits unrestricted, non-commercial use, distribution and reproduction in any medium, provided the work is properly cited. 